See discussions, stats, and author profiles for this publication at: https://www.researchgate.net/publication/271788998

\title{
Collision Free Control of Variable Length Hyper Redundant Robot Manipulator
}

Article · March 2014

DOI: 10.4028/www.scientific.net/AMM.541-542.1107

CITATION

1

7 authors, including:

Annisa Jamali

University Malaysia Sarawak

22 PUBLICATIONS 49 CITATIONS

SEE PROFILE

(2)

Md Mozasser Rahman

International Islamic University Malaysia

24 PUBLICATIONS 142 CITATIONS

SEE PROFILE
READS

17

Md Raisuddin Khan

International Islamic University Malaysia

93 PUBLICATIONS 261 CITATIONS

SEE PROFILE

Mohd Syahmi Jamaludin

University Malaysia Sarawak

12 PUBLICATIONS 16 CITATIONS

SEE PROFILE

Some of the authors of this publication are also working on these related projects:

Mechatronics Systems View project

Project Gas powered firefighting robot applications View project 


\title{
Collision Free Control of Variable Length Hyper Redundant Robot Manipulator
}

\author{
Annisa Jamali ${ }^{1, \mathrm{a}}$, M. Raisuddin Khan ${ }^{2, \mathrm{~b}}$, M. Shahril Osman ${ }^{1, \mathrm{c}}$, \\ M. Mozasser Rahman ${ }^{2, \mathrm{~d}}$, Muhd Fadzli Ashari ${ }^{1, \mathrm{e}}$, Mohd Syahmi Jamaludin ${ }^{1, \mathrm{f}}$ and \\ Ervina Junaidi ${ }^{1, g}$ \\ ${ }^{1}$ Department of Mechanical and Manufacturing,Universiti Malaysia Sarawak, \\ Kota Samarahan-Kuching Expressway, Sarawak, Malaysia. \\ ${ }^{2}$ Department of Mechatronics Engineering, International Islamic University Malaysia, \\ Jalan Gombak, Kuala Lumpur, Malaysia. \\ ajannisa@feng.unimas.my
}

Keywords: Robot manipulator, hyper-redundant robot, path planning, collision free, Introduction

\begin{abstract}
Hyper-redundant robot (HRR) manipulators are useful at navigating convoluted paths, but conventionally complicated to control. The control of a hyper-redundant manipulator is complex due to its redundancy. In this paper, a simple but effective control algorithm for obstacle avoidance is proposed. The algorithm derives a collision free path around known obstacles so that the end-effector of a variable length hyper redundant robot (VHRR) is able to reach the target location following the path without hitting the obstacles. The algorithm can be grouped into two tasks to drive the endeffector along the collision free trajectories: first, solve the inverse kinematics without disregarding the existence of obstacles in the system; and second, fit the manipulator to the respective prescribe trajectories. This method has the capability to allow VHRR maneuver within its workspace without penetrating to the neighboring obstruction. Further, this method is very effective in the sense that it forms a nice coil profile avoiding zig-zag configuration, and thus eliminates sharp turn on the robot. The performance of a VHRR was tested through simulation to demonstrate the effectiveness of the proposed method. The approach succeeded in delivering the path that avoids obstacle.
\end{abstract}

\section{Introduction}

Redundant manipulators present their potential abilities from theoretical aspect as well as practical aspect, thus, has attracted a lot of attention. The capability includes a fundamental problem to generate collision free path of static or moving obstacles in the workspace. Generally, there are two main strategies to tackle the problem of collision free maneuvers namely global planning and local control approach. The global planning is associated with high-level path planning in which the collision free path is mapped off-line, thus, guaranteed to find the unoccupied workspace [1]-[2]. In the contrary, the local control is carried out on-line based on information provided by the sensors, thus, the obstacle position is detected in the real time during the task execution [3-5]. The local control approach allows flexibility in the control system. However, the flexibility offered may cause the robot to stall when the path cannot be identified. Thus, it gives a significant advantage to control this mechanism by a predefined set of motions via global planning due to a great deal of redundancy rather than to calculate their route in the real time.

There are several approaches introduced to solve the inverse kinematics which may distinguish into three main group that is the algebraic [6] (e.g. Nonlinear optimization, Jacobian pseudo inverse and its optimization), evolutionary computation [7-8] (e.g. Artificial Neural Network, Genetics Algorithm, ANFIS, Fuzzy Logic) and geometric approach [9-10]. The major shortcoming of the algebraic and iterative technique is that there is no proper indication for choosing the best solution out of numerous solutions available for the n-number of links, and the derivation of the equation becomes more complicated when the level of redundancy increases.

The control scheme designed in this research is applicable to both HRR and VHRR giving priorities to collision free path around obstacles. The geometric approach took a complete departure from the existing methods and offered substantially good accuracy and avoided the computation of 